IFT - P.004/98

hep-ph/9712503

\title{
The action for the (propagating) torsion and the limits on the torsion parameters from present experimental data
}

\author{
A.S.Belyaev a,b,円, Ilya L.Shapiro ${ }^{c, d, 2 ~}$ \\ a Instituto de Física Teórica, Universidade Estadual Paulista, \\ Rua Pamplona 145, 01405-900 - São Paolo, S.P., Brasil \\ b Skobeltsyn Institute of Nuclear Physics, Moscow State University, \\ 119 899, Moscow, Russian Federation \\ c Departamento de Fisica, Universidade Federal de Juiz de Fora, 36036-330, MG - Brazil \\ d Tomsk State Pedagogical University, 634041, Tomsk, Russia
}

\begin{abstract}
Starting from the well established form of the Dirac action coupled to the electromagnetic and torsion field we find that there is some additional softly broken local symmetry associated with torsion. This symmetry fixes the form of divergences of the effective action after the spinor fields are integrated out. Then the requirement of renormalizability fixes the torsion field to be equivalent to some massive pseudovector and its action is fixed with accuracy to the values of coupling constant of torsion-spinor interaction, mass of the torsion and higher derivative terms. Implementing this action into the abelian sector of the Standard Model we establish the upper bounds on the torsion mass and coupling. In our study we used results of present experimental limits on four-fermion contact interaction (LEP, HERA, SLAC, SLD, CCFR) and TEVATRON limits on the cross section of new gauge boson, which could be produced as a resonance at high energy $p \bar{p}$ collisions.
\end{abstract}

\footnotetext{
${ }^{1}$ e-mail: belyaev@ift.unesp.br

${ }^{2}$ e-mail: shapiro@ibitipoca.fisica.ufjf.br
} 


\section{Introduction}

The great success of the Standard Model (SM) in the predictions of the results of experiments on the accelerators is very impressive. On the other hand the common point of view is that SM and its direct generalizations such as GUT's can not serve as a fundamental theory at a very high energies because they do not include quantum gravity. These theories should be regarded as an effective field theories which are only valid at the restricted energy range [1]. If the fundamental "ultimate" theory will be someday achieved, it will probably differ from the conventional quantum field theory and include the nonlocal implications. As an example of such a theory one can consider the string theory which produce gravity as an induced interaction and thus solves the quantum gravity problem. In fact the construction of mathematically consistent string theory (or its generalizations) is only part of the work that has to be done. The next problem is to understand in which way the unified theory can manifest itself.

One has to notice that the consistent string theory predicts, along with the metric, other components of the gravitational field. In particular, the antisymmetric second rank field $A_{\alpha \beta}$ enters the string effective action via its antisymmetrized derivatives $T_{\alpha \beta \gamma}=\partial_{[\alpha} A_{\beta \gamma]}$ [2] which are usually referred to as the antisymmetric torsion field. Therefore the torsion field is predicted by string theory and it is quite interesting to establish what the effects of torsion can look like. Recently there were an interesting works devoted to this problem. In particular, the effects of external background torsion to the quantized matter fields were discussed in [3, 4, 5, 6, 7, 8, 9, 10, 11], and some of these papers [7, 10 contain numerical bounds on the possible torsion effects. The phenomenological consequences of the propagating torsion were considered in [12]. The first of this papers contains an interesting discussions in the contest of effective field theory and some upper bounds for torsion. However in this paper the torsion is regarded as a pseudoscalar longitudinal mode of the antisymmetric tensor while the vector transversal mode was neglected. The purpose of this letter is to prove that the action of dynamical torsion necessary contains massive vector field and to evaluate its possible observational consequences. We derive the action for the torsion pseudovector and find that it contains two free parameters (one of them is torsion mass) and study the phenomenological consequences of this action. As the result of this study we obtain an upper bounds on the parameters of the torsion action using present experimental data.

The paper is organized in the following way. In the next section we introduce a basic notations, give a very brief review of gravity with torsion and establish an additional softly broken symmetry which holds for the spinor field coupled to torsion (see also [11]). Then we discuss the possible form of divergences which may appear in the theory with propagating torsion and find the form of the action which provides the renormalizability. Section 3 is devoted to the phenomenological consequences of the propagating torsion, and in section 4 we draw some conclusions and outline the perspectives for the future study.

\section{Spinor field coupled to torsion and electromagnetic field}

Let us give a very brief review for the basical notions of the gravity with torsion. One can find more detailed introduction to the gravity with torsion in Refs. [13, 14] and to the quantum field theory in curved space-time with torsion in [15, which notations we use below. The metric $g_{\mu \nu}$ and torsion $T_{\beta \gamma}^{\alpha}$ have to be considered as an independent characteristics of the space - time. Since here we are interested in the torsion effects only, the metric is supposed to be flat Minkowski one everywhere. In the theory with torsion the covariant derivative $\tilde{\nabla}$ 
is based on the nonsymmetric connection $\tilde{\Gamma}_{\beta \gamma}^{\alpha}$ with

$$
\tilde{\Gamma}_{\beta \gamma}^{\alpha}-\tilde{\Gamma}_{\gamma \beta}^{\alpha}=T_{\beta \gamma}^{\alpha},
$$

Indeed $\tilde{\Gamma}_{\beta \gamma}^{\alpha}$ is not a tensor because one can use the curvilinear coordinates. The metricity condition $\tilde{\nabla}_{\mu} g_{\alpha \beta}=0$ enables one to express the connection through metric and torsion in a unique way as

$$
\tilde{\Gamma}_{\beta \gamma}^{\alpha}=\left\{\begin{array}{c}
\alpha \\
\beta \gamma
\end{array}\right\}+\frac{1}{2}\left(T_{\beta \gamma}^{\alpha}-T_{\beta \cdot \gamma}^{\alpha}-T_{\gamma \cdot \beta}^{\alpha}\right)
$$

where $\left\{\begin{array}{c}\alpha \\ \beta \gamma\end{array}\right\}$ is the Christoffel symbol. It is important that the rest of the formula (2) is tensor and thus it can not be eliminated by the change of coordinates. It proves convenient to divide the torsion field into three irreducible components: Trace $T_{\beta}=T_{\beta \alpha}^{\alpha}$; Pseudotrace $S^{\nu}=\epsilon^{\alpha \beta \mu \nu} T_{\alpha \beta \mu}$ and the tensor $q_{\beta \gamma}^{\alpha}$, for which the two conditions are satisfied

$$
q_{\beta \alpha}^{\alpha}=0 ; \quad \epsilon^{\alpha \beta \mu \nu} q_{\alpha \beta \mu}=0 .
$$

In the string-induced action, which depends on the completely antisymmetric torsion, only the pseudovector part $S_{\mu}$ is present, and thus one can always set

$$
T_{\alpha \beta \mu}=\frac{1}{6} \varepsilon_{\alpha \beta \mu \nu} S^{\nu}
$$

Below we shall use the pseudovector $S_{\mu}$ as parametrization for the antisymmetric torsion tensor.

The minimal action of the Dirac spinor fields in an external gravitational field with torsion follows from the standard procedure (see, for example, [15]). One has to change the partial derivatives $\partial_{\mu}$ to the covariant ones $\tilde{\nabla}_{\mu}$. In case of the general Riemann-Cartan manifold [13, 15] one has also to change the flat metric $\eta^{\mu \nu}$ to the general one $g^{\mu \nu}$ and generalize the volume element $d^{4} x$ to the covariant one $d^{4} x \sqrt{-g}$. In our case $g_{\mu \nu}=\eta_{\mu \nu}$ this procedure leads to the expression:

$$
\begin{gathered}
S_{\frac{1}{2}, \min }=\frac{i}{2} \int d^{4} x\left(\bar{\psi} \gamma^{\alpha} \tilde{\nabla}_{\alpha} \psi-\tilde{\nabla}_{\alpha} \bar{\psi} \gamma^{\alpha} \psi-2 i m \bar{\psi} \psi\right) \\
=i \int d^{4} x \bar{\psi}\left(\gamma^{\alpha} \partial_{\alpha}-\frac{i}{8} \gamma_{5} \gamma^{\alpha} S_{\alpha}-i m\right) \psi
\end{gathered}
$$

Here $\bar{\nabla}$ is covariant derivative of the spinor field (see [15] for the details.)

The renormalizability of the gauge model in an external torsion field requires the nonminimal interaction of the spinor and scalar fields with torsion [3]. Later on we shall see that this is reasonable to introduce the nonminimal spinor-torsion interaction in the theory with the propagating torsion as well. For this reason we shall start our study from the action of the Dirac spinor nonminimally coupled with the electromagnetic and torsion fields

$$
S_{1 / 2}=i \int d^{4} x \sqrt{-g} \bar{\psi}\left[\gamma^{\alpha}\left(\partial_{\alpha}-i e A_{\alpha}+i \eta \gamma_{5} S_{\alpha}\right)-i m\right] \psi
$$

The new interaction with torsion doesn't spoil the invariance of the above action under usual gauge transformation:

$$
\psi^{\prime}=\psi e^{\alpha(x)}, \quad \bar{\psi}^{\prime}=\bar{\psi} e^{-\alpha(x)}, \quad A_{\mu}^{\prime}=A_{\mu}-e^{-1} \partial_{\mu} \alpha(x)
$$


Moreover the massless part of the action (5) is invariant under the transformation in which the pseudotrace of torsion plays the role of the gauge field

$$
\psi^{\prime}=\psi e^{\gamma_{5} \beta(x)}, \quad \bar{\psi}^{\prime}=\bar{\psi} e^{\gamma_{5} \beta(x)}, \quad S_{\mu}^{\prime}=S_{\mu}-\eta^{-1} \partial_{\mu} \beta(x)
$$

Thus in the massless sector of the theory one faces generalized gauge symmetry depending on scalar $\alpha(x)$ and pseudoscalar $\beta(x)$ parameters of transformation. As it will be shown below, this new symmetry requires torsion to be massive vector field and furthermore the action of torsion is fixed with accuracy to the values of nonminimal parameter $\eta$, mass of the torsion $M_{t s}$ and possible higher derivative terms.

In the framework of effective field theory the effects of a very massive fields are suppressed by the factors of $\mu^{2} / M^{2}$ where $M$ is the mass of the field and $\mu$ the typical energy of the process. Thus if we take $M_{t s}$ to be of the Planck order then the effects of torsion will be negligible at the energies available at the modern experimental facilities. The hypothesis of torsion, propagation at energies lower than the Planck one supposes that $M$ is essentially smaller than the Planck mass. Then we have two options: take torsion to be massless or consider the mass of torsion as a free parameter which should be defined on the experimental basis. As far as the torsion can propagate, one has to incorporate it into the SM along with other vector fields. Let us discuss the form of the action for torsion, which leads to the consistent quantum theory. The higher derivative terms in the action, in general, lead to the unphysical ghosts and to the consequent violation of unitarity. Therefore we restrict the torsion action by the second derivative and zero-derivative terms. The general action including these terms has the following form:

$$
S_{\text {tor }}=\int d^{4}\left\{-a S_{\mu \nu} S^{\mu \nu}+b\left(\partial_{\mu} S^{\mu}\right)^{2}+M_{t s}^{2} S_{\mu} S^{\mu}\right\}
$$

where $S_{\mu \nu}=\partial_{\mu} S_{\nu}-\partial_{\mu} S_{\nu}$ and $a, b$ are some positive parameters. The action (\$) contains both transversal vector mode and the longitudinal model which is in fact equivalent to the scalar (see, for example, discussion in [12]) In particular, in the $a=0$ case only the scalar mode, and for $b=0$ only the vector mode propagate. It is well known 17 that in the unitary theory of the vector field both longitudinal and transversal modes can not propagate, and therefore, in order to have consistent theory of torsion one has to choose one of parameter $a, b$ to be zero 尚.

In fact the only correct choice is $b=0$. To see this one has to reveal that the symmetry, which is spoiled by the massive terms only, is always preserved in the renormalization of the dimensionless couplings constants of the theory. In other words, the divergences and corresponding local counterterms, which produce the dimensionless renormalization constants, do not depend on the dimensional parameters such as the masses of the fields. The symmetry (7) holds for the massless part of the action (5) and therefore on the general grounds one has to expect that the gauge invariant counterterm $\int S_{\mu \nu}^{2}$ appears if we take the loop corrections into account.

We want emphasize that in the framework of effective field theory the level of approximation for taking into account the massive fields is qualitatively the same for the tree level and for the lower loop effects. Therefore as far as the propagating torsion is considered and

\footnotetext{
${ }^{3}$ This kind of torsion equivalent to the pseudoscalar field was introduced in [16] in order to maintain the gauge invariance of abelian vector field in the Riemann-Cartan spacetime.

${ }^{4}$ We remark that earlier the unitarity was effectively used for the construction of the action of gravity with torsion in 18, 19.
} 
the kinetic term in (8) is taken into account, one has to formulate the theory as renormalizable. Neglecting the high energy effects while the low energy amplitudes are considered may mean that we disregard some higher derivative terms. However the violation of the renormalizability in that sectors of the theory which are taken into account is impossible. For instance, if we start from the purely scalar longitudinal torsion (as the authors of [12] did) then the transversal term $\int S_{\mu \nu}^{2}$ will arise with the divergent coefficient and this will indeed violate both the finiteness of the effective action and the unitarity of the $S$-matrix. All this is true even in the case that only the tree-level effects are evaluated, if only such consideration is regarded as an approximation to any reasonable quantum theory.

Thus the kinetic term of the torsion action is given by the Eq. (8) with $b=0$. As concerns the massive term it is not forbidden by the symmetry (7), because the last is softly broken. Therefore apriory there are no reasons to suppose that $M_{t s}=0$. The only one question is: whether the massive counterterm really appears if we take into account the fermion loops. To investigate this we have performed the one-loop calculation of divergences in the theory (5), using the standard Schwinger-deWitt technique and dimensional regularization (one can see [15] for introduction and references). The result of these calculations of

$$
\Gamma_{d i v}[A, S]=-\operatorname{Tr} \ln \left[i \gamma^{\alpha}\left(\partial_{\alpha}-i e A_{\alpha}+i \eta \gamma_{5} S_{\alpha}\right)-i m\right]_{d i v}
$$

Is the following counterterm:

$$
\Delta S\left[A_{\mu}, S \alpha\right]=\frac{1}{\varepsilon} \int d^{4} x\left\{\frac{2 e^{2}}{3} F_{\mu \nu} F^{\mu \nu}+\frac{2 \eta^{2}}{3} S_{\mu \nu} S^{\mu \nu}-\frac{i e \eta}{3} \epsilon^{\alpha \beta \mu \nu} S_{\mu \nu} F_{\alpha \beta}+8 m^{2} \eta^{2} S^{\mu} S_{\mu}\right\}
$$

with $\varepsilon=(4 \pi)^{2}(n-4)$. Here we have neglected all surface terms except $\epsilon^{\alpha \beta \mu \nu} S_{\mu \nu} F_{\alpha \beta}$, because it can, in principle, lead to quantum anomaly. The phenomenological consideration below is restricted by the tree-level effects and therefore this term is beyond the scope of our present interest. The form of the counterterms (10) indicates that the massive term in the action of torsion is indeed necessary for the renormalizability and hence the correct form of the action is

$$
S_{\text {tor }}=\int d^{4}\left\{-\frac{1}{4} S_{\mu \nu} S^{\mu \nu}+M_{t s}^{2} S_{\mu} S^{\mu}\right\}
$$

In the last expression we put the conventional coefficient $-1 / 4$ in front of the kinetic term. With respect to the renormalization this means that we (in a direct analogy with QED) can remove the kinetic counterterm by the renormalization of the field $S_{\mu}$ and then renormalize the parameter $\eta$ in the action (5) such that the combination $\eta S_{\mu}$ is the same for the bare and renormalized quantities. Instead one can include $1 / \eta^{2}$ into the kinetic term of (11), that should lead to the direct renormalization of this parameter while the interaction of torsion with spinor has minimal form (何) and $S_{\mu}$ is not renormalized. Therefore in the case of propagating torsion the difference between minimal and nonminimal interactions is only the question of notations on both classical and quantum levels.

In the next section we shall discuss the possible consequences of the torsion action at low energies and find some numerical upper bounds for torsion. From the string theory point of view $M_{t s}$ should have the value of the Planck order. Indeed this choice doesn't

\footnotetext{
${ }^{5}$ The same calculation for the massless theory in curved space-time has been performed in 20 (see also references there and in hep-th version of [5]). More details about (10) and consequent renormalization group equations will be given elsewhere 21.
} 
give a chance to make any speculations and estimates using an available experimental data because they are obtained at the energies which are (at least) 16 orders smaller than the Planck ones. Therefore we suppose that as a result of some cancelation the string inspired mass $M_{t s}$ vanishes and then take $M_{t s}$ to be some free parameter of the theory. We shall consider two different possibilities: i) torsion is much more heavy than other particles of SM and ii) torsion has a mass comparable to that of other particles. In the last case one meets a propagating particle which must be treated on an equal footing with other constituents of SM. Contrary to that, the very heavy torsion leads to the effective contact four-fermion interactions.

Consider this in some more details, starting from the actions (5) and (11). Since the massive term dominates over the covariant kinetic part of the action, the last can be disregarded. Then the action $S_{1 / 2}+S_{\text {tor }}$ leads to the algebraic equation of motion for $S_{\mu}$. The solution of this equation can be substituted back to $S_{1 / 2}+S_{\text {tor }}$ and thus produce the contact four-fermion interaction term

$$
\mathcal{L}_{i n t}=-\frac{\eta^{2}}{M_{t s}^{2}}\left(\bar{\psi} \gamma_{5} \gamma^{\mu} \psi\right)\left(\bar{\psi} \gamma_{5} \gamma_{\mu} \psi\right)
$$

As one can see the only one quantity which appears in this approach is the ratio $M_{t s} / \eta$ and therefore the phenomenological consequenses may depend only on single parameter.

In the next section we consider the upper bounds on the phenomenological manifestations of contact interactions between quarks and leptons and also some observational limits for the propagating torsion.

\section{Possible physical observables related with torsion action and the limits on the torsion parameters}

In this section we put the limits on the parameters of the torsion action using results of various experiments ? Torsion being a pseudo-vector particle interacting with fermions might give therefore different physical observables.

Physical observables related with torsion depend on the two basic parameters, namely on the torsion mass $M_{t s}$ and the constant of the interaction between torsion and fermion fields $\eta$. In the course of our study we choose, for the sake of simplicity, all the torsion couplings with fermions to be the same $\eta$ [21]. This enables one to put the limits in the two dimensional $\left(M_{t s}-\eta\right)$ parameter space using the present experimental data.

The straightforward consequence of the action term for torsion interaction with fermion field is the effective four-fermion contact interaction of leptons and quarks (12). Four-fermion interaction effectively appears for the torsion with mass much higher than the energy scale available at present colliders. There are several experiments from which the constraints on the contact four-fermion interactions come:

1)Experiments on polarized electron-nucleus scattering - SLAC e-D scattering experiment 22, Mainz e-Be scattering experiment [23] and bates e-C scattering experiment [24];

2)Atomic physics parity violations measures [25] electron-quark coupling that are different from those tested at high energy experiment provides alternative constraints on new physics. 3) $e^{+} e^{-}$experiments - SLD, LEP1, LEP1.5 and LEP2 (see for example [26, 27, 28, 29, 30]); 4)Neutrino-Nucleon DIS experiments - CCFR collaboration obtained model independent constraint on the effective $\nu \nu q q$ coupling [31];

\footnotetext{
${ }^{6}$ The detailed exposition will be presented elsewhere 21
} 
5)HERA experiment for the polarized lepton-proton beams interactions [32].

First we consider a limits on the contact interactions induced by torsion. The contact four-fermion interaction may be described by the Lagrangian [34] of the most general form:

$$
L_{\psi^{\prime} \psi^{\prime} \psi \psi}=g^{2} \sum_{i, j=L, R} \sum_{q=u, d} \frac{\epsilon_{i j}}{\left(\Lambda_{i j}^{\epsilon}\right)^{2}}\left(\bar{\psi}_{i}^{\prime} \gamma_{\mu} \psi_{i}^{\prime}\right)\left(\bar{\psi}_{j} \gamma^{\mu} \psi_{j}\right)
$$

Subscrips i,j refer to different fermion helicities: $\psi_{i}^{\left({ }^{(}\right)}=\psi_{R, L}^{\left({ }^{\prime}\right)}=\left(1 \pm \gamma_{5}\right) / 2 \cdot \psi^{\left({ }^{\prime}\right)}$; where $\psi^{\left({ }^{\prime}\right)}$ could be quark or lepton; $\Lambda_{i j}$ represents the mass scale of the exchanged new particle; coupling strength is fixed by the relation: $g^{2} / 4 \pi=1$, the sign factor $\epsilon_{i j}= \pm 1$ allows for either constructive or destructive interference with Standard Model (SM) $\gamma$ and $Z$-boson exchange amplitudes. The formula (13) can be successfully used for the study of the torsion-induced contact interactions because it includes an axial-axial current interactions as a particular case.

Recently the global study of the electron-electron-quark-quark(eeqq) interaction sector of the SM [33] have been done using data from all mentioned above experiments (except the fifth item). The limits established in this paper are the best in comparison with the previous ones. Since the effective contact Lagrangian for torsion has axial-axial structure we used the limits obtained in paper [33] for this kind of interaction. We remark that the limits on $\Lambda$ of paper [32] are quite close to those of [33 but are not suitable for our analysis because axial-axial interaction have not been studied in [32]. Axial-axial current may be expressed through RR,LR,RL and LR currents in the following way:

$$
j_{\mu}^{A} j_{\mu}^{A}=\frac{j_{\mu}^{L} j_{\mu}^{L}+j_{\mu}^{R} j_{\mu}^{R}-j_{\mu}^{L} j_{\mu}^{R}-j_{\mu}^{R} j_{\mu}^{L}}{4} .
$$

For the axial-axial eeqq interactions (13) takes the form (we put $g^{2}=4 \pi$ ):

$$
L_{e e q q}=-\frac{4 \pi}{\left(\Lambda_{A A}^{\epsilon}\right)^{2}}\left(\bar{e} \gamma_{\mu} \gamma_{5} e\right)\left(\bar{q} \gamma^{\mu} \gamma_{5} q\right)
$$

The limit for the contact axial-axial eeqq interactions comes from the global analysis of Ref. [33]:

$$
\frac{4 \pi}{\Lambda_{A A}^{2}}<0.36 \mathrm{TeV}^{-2}
$$

For the parameters of the effective contact four-fermion interactions of general form (13) and contact four fermion interactions induced by torsion (12) we have the following relations:

$$
\frac{\eta^{2}}{M_{t s}^{2}}=\frac{4 \pi}{\Lambda_{A A}{ }^{2}}
$$

¿From (16) and (14) one gets the following limit on torsion parameters:

$$
\frac{\eta}{M_{t s}}<0.6 \mathrm{TeV}^{-1} \Rightarrow M_{t s}>1.7 \mathrm{TeV} \cdot \eta
$$

The limits on $M_{t s}$ and $\eta$ coming from the (18) is shown in Figure 1(A). Some remark about the energy limits taken in this plot is in order. We started exclusion region from $M_{t s}=1 \mathrm{TeV}$. This choice is related with the fact that the application of effective-contact interactions (12) is valid up to the certain mass of the torsion below which an exact calculation (regarding the 

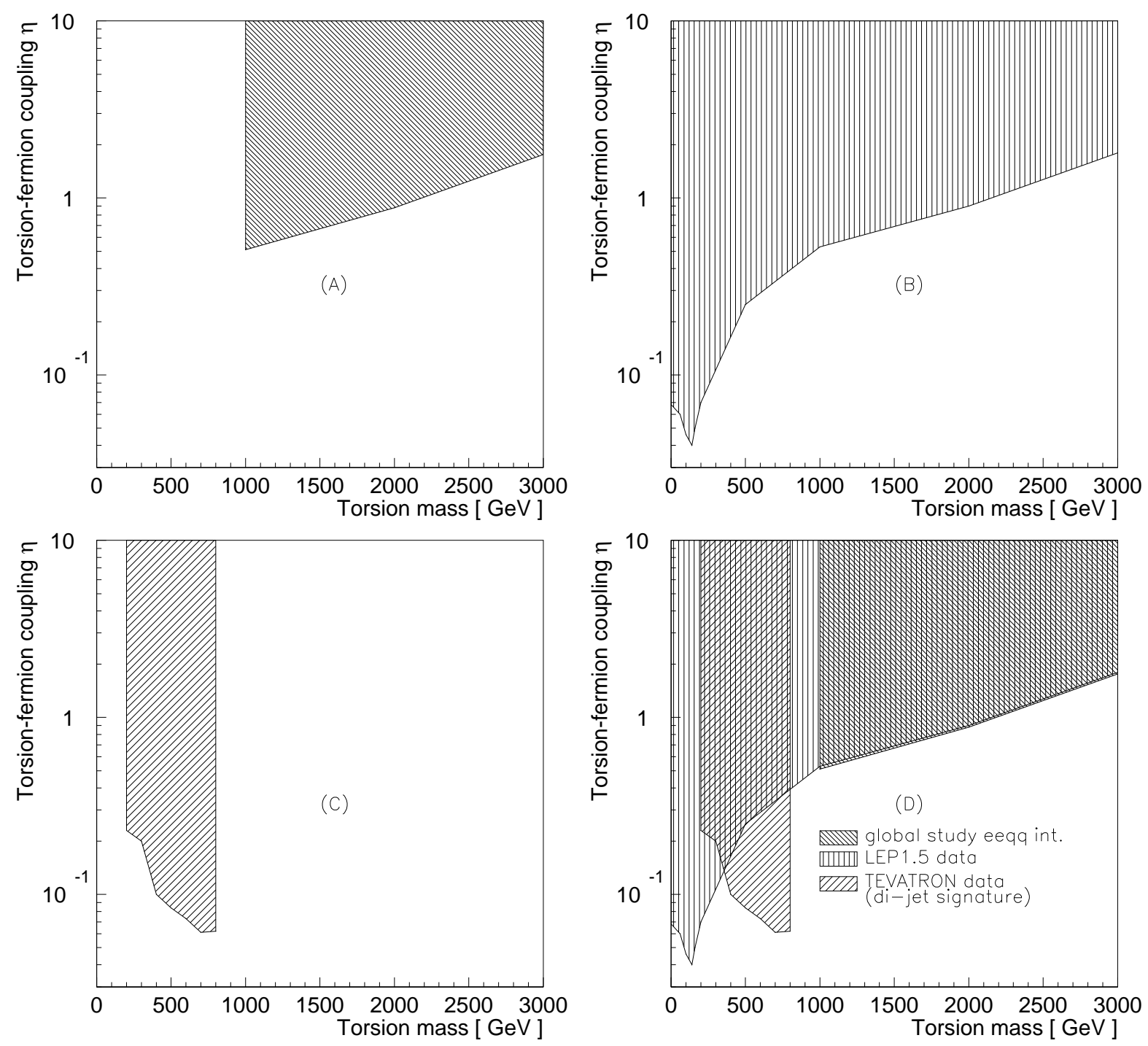

Figure 1: Allowed regions for $M_{t s}$ and $\eta$ coming from global study of electron-quark contact interactions (A), LEP1.5 (B) and TEVETRON data (C). (D) - combined limit. Hatched region is excluded by experiments mentioned above

field $S_{\mu}$ as dynamical) should be done. The relative data of the two approaches are shown on the Figure 2, where the results for gauge interaction (11) and contact interactions (13) for torsion are compared. As an example we have calculated total cross section for LEP1.5 with $\sqrt{s}=140 \mathrm{GeV}$ and $\eta$ equal to 0.5 . One can clearly see that for torsion heavier than $1 \mathrm{TeV}$ the approximation of the effective contact interaction works almost perfectly, reproducing the result for the exact calculation with $0.1 \%$ accuracy. Therefore the scale $1 \mathrm{TeV}$ is appropriate starting point for putting the limit on torsion parameters using the Lagrangian with contact interactions. Scenario with light torsion is in general more difficult because here we have two independent parameters and thus are enforced to study the 2-dimensional restrictions from the experimental data. Indeed there is no rigid border between two cases, as we shall see below.

For constraining $M_{t s}-\eta$ parameter space for light torsion we use results of LEP1.5 analysis of paper [27]: the cross section of $e^{+} e^{-} \rightarrow e^{+} e^{-}\left(\mu^{+} \mu^{-}\right)$process was measured with 


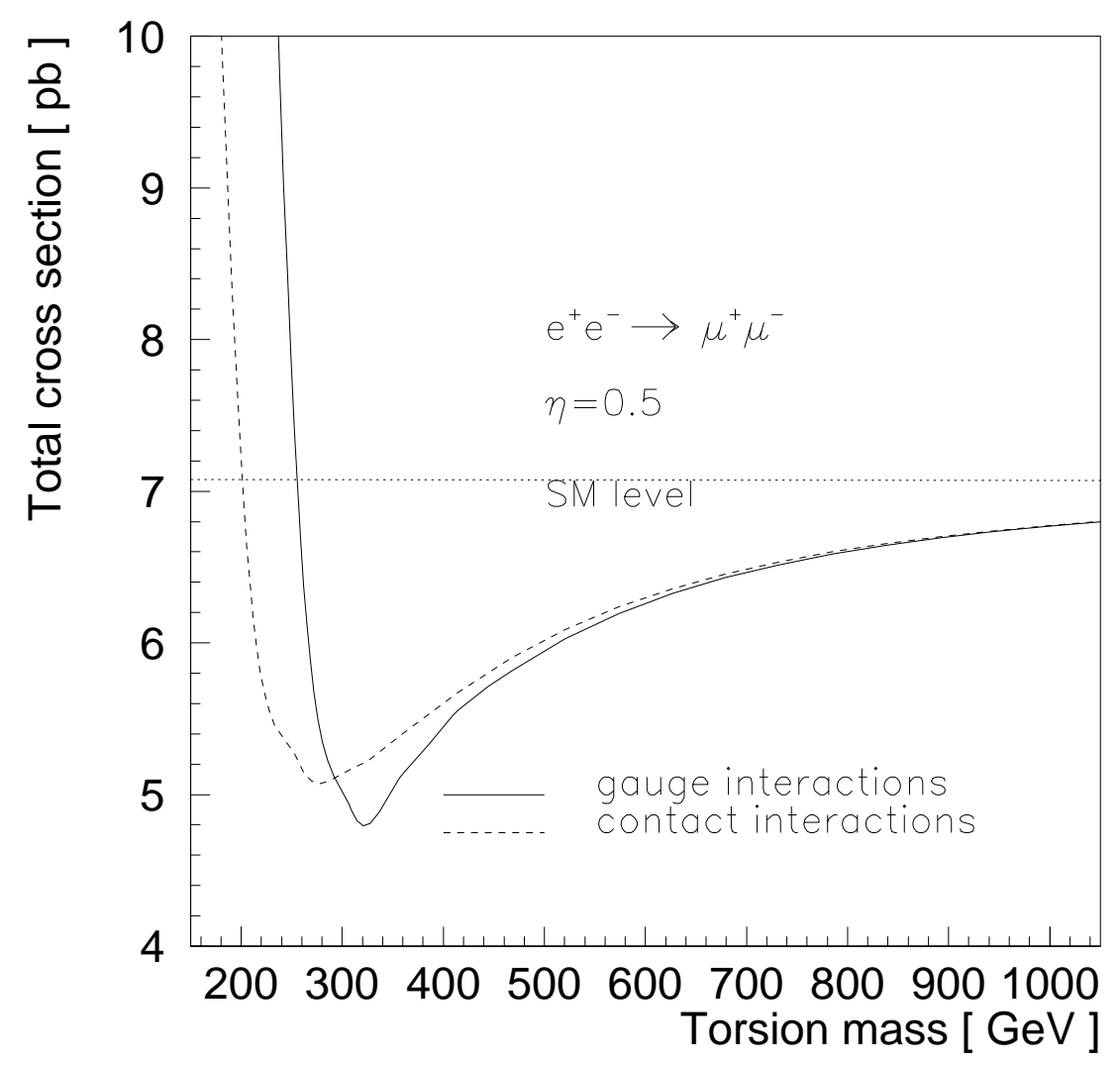

Figure 2: Comparison of the total cross sections of $e^{+} e^{0} \rightarrow \mu^{+} \mu^{-}$process for gauge and contact interactions

accuracy 1-2\%. We used this fact to put the limits on torsion mass and coupling: $90 \%$ acceptance for electron and $60 \%$ for muon channels was assumed, the total cross section for these reaction were calculated and $4 \%$ deviation from the Standard Model prediction was taken for establishing the limits. The results constraints is shown in Figure 1(B).

The torsion with the mass in the range of present colliders could be produced in fermionfermion interactions as a resonance, decaying to fermion pair. The most promising collider for search the signature of such type is TEVATRON. Search for New Particles Decaying to two-jets has been done recently by D0 and CDF collaborations [35]. The data that we use in our analysis are extracted from figure presented D0 collaboration which established the limit on the production cross section of $Z^{\prime}$ and $W^{\prime}$ bosons . Here we assume also $90 \%$ events efficiency (including efficiency of kinematical cuts and trigger efficiency) and calculated the cross section for torsion production at TEVATRON. Then we applied D0 limit at 95\% CL for torsion production cross section and converted into the limit for $M_{t s}-\eta$ plane. This limit is shown in figure $1(\mathrm{C})$. The points for the exclusion curve are given in Table 1.

\begin{tabular}{l|l|l|l|l|l|l|l}
$M_{t s}(\mathrm{GeV})$ & 200 & 300 & 400 & 500 & 600 & 700 & 800 \\
\hline$\eta$ & 0.23 & 0.20 & 0.10 & 0.084 & 0.073 & 0.061 & 0.062
\end{tabular}

Table 1: Points for exclusion curve in $M_{t s}-\eta$ plane from TEVATRON data

One can see that the limits on $\eta$ coming from these analysis are much better in comparison with those from the LEP data. Combined exclusion plot for $M_{t s}-\eta$ plane is presented in 
Fig. 1(D).

It should be stressed that all numerical and symbolic calculations for establishing limits on torsion parameter space have been done using CompHEP software package [36] where the torsion action was introduced.

\section{Conclusions}

We derived the action of propagating torsion and implemented it into the abelian sector of the Standard Model. It was shown that the only one action of torsion which leads to consistent (unitary and renormalizable) theory includes propagating pseudovector massive particle with softly broken (new) gauge symmetry. Starting from this action for torsion, we have established some upper bounds on the torsion mass and torsion-spinor coupling constant (which is supposed to be universal) using combined limit for four-fermion interactions, LEP and TEVATRON data. For heavy torsion the limit is described by relation (18) while for light torsion with the mass below $1 \mathrm{TeV}$ limits coming from LEP and TEVATRON data bound $\eta$ to be less than 0.1-0.02 depending on $M_{t s}$. We are going to give more details about these results in future publication [21].

Another interesting aspect concerns the possibility to implement scalar fields. The study of the GUT-like theories with Yukawa scalar-spinor interactions in an external torsion field [3] has demonstrated the necessity to introduce the nonminimal interaction of scalar fields with torsion. When one is considering the propagating torsion, all the diagrams which were considered in [3] still give the same contributions and therefore it is very probable that the full consistent theory contains a nonminimal scalar-torsion interaction and also the torsion self-interaction term. These interaction will not change significantly observables considered in this paper since the physical effects from scalar-torsion interaction and also the torsion self-interaction will only appear at loop level. But they might lead to some other physical observables which probably could allow us to improve the limits on the torsion parameters. Moreover some formal questions related with the torsion self-interaction can be addressed. We hope to be back to these problems in the close future.

\section{Acknowledgments}

One of the authors (I.L.Sh.) is grateful to M. Asorey, I.L. Buchbinder, I.B. Khriplovich and T. Kinoshita for stimulating discussions. He also acknowledges warm hospitality of Departamento de Fisica, Universidade Federal de Juiz de Fora and partial support by Russian Foundation for Basic Research under the project No.96-02-16017. A.S.B. acknowledges support from Fundação de Amparo à Pesquisa do Estado de São Paulo (FAPESP). 


\section{References}

[1] S. Weinberg, The Quantum Theory of Fields: Foundations. (Cambridge Univ. Press, 1995).

[2] M.B. Green, J.H. Schwarz and E. Witten, Superstring Theory (Cambridge University Press, Cambridge, 1987).

[3] I.L. Buchbinder and I.L. Shapiro, Phys.Lett. 151B (1985) 263.

[4] I.L. Buchbinder and I.L. Shapiro, Class. Quantum Grav. 7 (1990) 1197; I.L. Shapiro, Mod.Phys.Lett.9A (1994) 729.

[5] V.G. Bagrov, I.L. Buchbinder and I.L. Shapiro, Izv. VUZov, Fisica (in Russian. (English translation: Sov.J.Phys.) 35,n3 (1992) 5 (see also at hep-th/9406122).

[6] R. Hammond, Phys.Lett. 184A (1994) 409; Phys.Rev. 52D (1995) 6918.

[7] R. Hammond, Class.Quant.Grav. 13 (1996) 1691.

[8] A. Dobado and A. Maroto, Phys.Rev. 54D (1996) 5185.

[9] A. Dobado and A. Maroto, Preprint hep-ph/9705434; Preprint hep-ph/9706044.

[10] C. Lammerzahl, Phys.Lett. 228A (1997) 223.

[11] L.H. Ryder and I.L. Shapiro, Paper in preparation.

[12] S.M. Caroll and G.B. Field, Phys.Rev. 50D (1994) 3867.

[13] F.W. Hehl, Gen. Relat.Grav.4(1973)333;5(1974)491; F.W. Hehl, P. Heide, G.D. Kerlick and J.M. Nester, Rev. Mod. Phys.48 (1976) 3641.

[14] "On the gauge aspects of gravity", F. Gronwald, F. W. Hehl, GRQC-9602013, Talk given at International School of Cosmology and Gravitation: 14th Course: Quantum Gravity, Erice, Italy, 11-19 May 1995, gr-qc/9602013

[15] I.L. Buchbinder, S.D. Odintsov and I.L. Shapiro, Effective Action in Quantum Gravity. (IOP Publishing - Bristol, 1992).

[16] M. Novello, Phys.Lett. 59A (1976) 105.

[17] L.D. Faddeev and A.A. Slavnov, Gauge fields. Introduction to quantum theory. (Benjamin/Cummings, 1980).

[18] D.E. Nevill, Phys.Rev. D18 (1978) 3535.

[19] E. Sezgin and P. van Nieuwenhuizen, Phys.Rev. D21 (1980) 3269.

[20] I.L. Buchbinder, S.D. Odintsov and I.L. Shapiro, Phys.Lett. 162B (1985) 92.

[21] A.S.Belyaev and I.L.Shapiro, Paper in progress.

[22] C.Y. Prescott et al., Phys. Lett. B84, 524 (1979) 
[23] W. Heil et al., Nucl. Phys. B327, 1 (1989)

[24] WP.A. Souder et al., Phys.Rev.Lett. 65, 694 (1990)

[25] P.Langasker, M.Luo and A.Mann, Rev.Mod.Phys. 64, 86 (1992)

[26] LEP Collaborations and SLD Collaboration, "A Combination of Preliminary Electroweak Measurements and Constrains on the Standard Model", prepared from contributions to the 28th International Conference on High Energy Physics, Warsaw, Poland, CERN-PPE/96-183 (Dec. 1996).

[27] OPAL Collaboration, G. Alexander et al., B391, 221 (1996).

[28] L3 Collaboration, Phys. Lett. B370, 195 (1996); CERN-PPE/97-52, L3 preprint 117 (May 1997).

[29] ALEPH Collaboration, Phys. Lett. B378, 373 (1996).

[30] P. Langacker and J. Erler, presented at the Ringberg Workshop on the Higgs Puzzle, Ringberg, Germany, 12/96, hep-ph/9703428.

[31] K.S. McFarland et al. (CCFR), FNAL-Pub-97/001-E, hep-ex/9701010.

[32] J.M. Virey, CPT-97-P-3542, To be published in the proceedings of 2nd Topical Workshop on Deep Inelastic Scattering off Polarized Targets: Theory Meets Experiment (SPIN 97), Zeuthen, Germany, 1-5 Sep 1997: Working Group on 'Physics with "Polarized Protons at HERA", hep-ph/9710423

[33] V. Barger, K. Cheung, K. Hagiwara, D. Zeppenfeld; MADPH-97-999, hep-ph/9707412. We are thankful to the authors of this paper for their analysis.

[34] E.Eichten, K.Lane, and M.Peskin, Phys.Rev.Lett. 50, 811 (1983).

[35] CDF Collaboration and D0 Collaboration (Tommaso Dorigo for the collaboration), FERMILAB-CONF-97-281-E, 12th Workshop on Hadron Collider Physics (HCP 97), Stony Brook, NY, 5-11 Jun 1997

[36] E.E.Boos, M.N.Dubinin, V.A.Ilyin, A.E.Pukhov, V.I.Savrin, SNUTP-94-116, INPMSU-94-36/358, hep-ph/9503280;

P.A.Baikov et al., Proc. of X Workshop on HEP and QFT (QFTHEP-95), ed. by B.Levtchenko, V.Savrin, p.101, hep-ph/9701412. 